\title{
The Role of Cytokine profile detection in Myelomatous pleural effusions
}

\author{
Junhui Xu \\ Peking University First Hospital \\ Liang Gao \\ Peking University First Hospital \\ Miao Yan \\ Peking University First Hospital \\ Bingjie Wang \\ Peking University First Hospital \\ Zhengyang Song \\ Peking University First Hospital \\ Huihui Liu \\ Peking University First Hospital \\ Kunyan Sun \\ Peking University First Hospital

\section{Lin Nong} \\ Peking University First Hospital \\ Mangju Wang \\ Peking University First Hospital \\ Xinan Cen ( $\nabla$ cenxn@bjmu.edu.cn ) \\ Peking University First Hospital
}

\section{Case report}

Keywords: Myelomatous pleural effusions, cytokine, INF-y, IL-2, immunomodulator

Posted Date: April 1st, 2021

DOI: https://doi.org/10.21203/rs.3.rs-363841/v1

License: () (1) This work is licensed under a Creative Commons Attribution 4.0 International License. Read Full License 


\section{Abstract}

Background: Myelomatous pleural effusion (MPE), as a presentation of extramedullary infiltration of multiple myeloma (MM), is rare and associated with poor outcomes without comparatively effective treatment now. The value of the cytokine detection in pleural effusions to MPE has not been reported at present.

Case presentation: We herein report a case of refractory and relapsed multiple myeloma which developed bilateral MPE due to disease progression caused by intolerance to various chemotherapy regimens. The cytomorphology and flow cytometry is adopted in the diagnosis confirmation. The chemotherapy containing immunomodulators combined with thoracic catheterization drainage is applied to the patient, showing a certain therapeutic effect. During the course of disease, the changes of cytokine profile in pleural effusion were monitored by Biolegend CBA technology, revealing that the cytokines such as IL- 6 and IL-10 related to the tumor load in pleural effusion decreased with the improvement of the disease, while IL-2, IL-4, IL-17A, TNF - a, INF - $\mathrm{Y}$, granzyme A, Granzyme B, perforin and granulysin increased with the improvement of the disease.

Conclusions: There is a prospect that the cytokines level in pleural effusion becomes an indication to evaluate treatment response of MPE, and in the light of our finding, immunomodulators, IL-2 and INF - $\gamma$ may be utilized in treating patients suffering MPE.

\section{Background}

MM is characterized by the abnormal proliferation of monoclonal plasma cells secreting large amounts of monoclonal immunoglobulin or light chains inside bone marrow and finally leads to anemia, hypercalcemia, bone destruction, kidney injury and so on. Incidence of pleural effusion in multiple myeloma is approximately $6 \%{ }^{1}$ whose causes include hypoalbuminemia, congestive heart failure caused by amyloidosis cardiomyopathy, renal dysfunction, pleural invasion etc. ${ }^{2}$ Among them we named the pleural effusion caused by pleural invasion as MPE, which is a type of extramedullary invasion with poor prognosis and treatment response. ${ }^{1}$ Other than tuberculous pleural effusion, the high level of ADA was also reported in 1 / 3 of MPE, which indicates MPE patients' changing immunocompetence because of ADA's important role in lymphatic system differentiation and maturation. ${ }^{3}$ Some cases reported that chemotherapy combined with intrathoracic injection of IL-2 or IFN - a brought a satisfying therapeutic effect, especially the former combination achieving an emission duration of up to 30 months. ${ }^{4,5}$ All these phenomena implied immune system played an important role in the mechanism of MPE. Therefore we applied Biolegend CBA technology to monitor cytokines in pleural effusion to explore the important role of cytokines during the disease course of a MPE patient.

\section{Case Presentation}

The subject was a 70-year-old female who visited Beijing Hospital in 2014 for weakness, amaurosis and profuse sweating. The hemoglobin concentration was $63 \mathrm{~g} / \mathrm{L}$. The white blood cell count was $6.49 \times 10^{9} / \mathrm{L}$. The platelet count was $100 \times 10^{9} / \mathrm{L}$. The laboratory chemistry profile showed total protein $110 \mathrm{~g} / \mathrm{L}$, albumin $36 \mathrm{~g} / \mathrm{L}$, calcium $2.32 \mathrm{mmol} / \mathrm{L}$, creatinine $74 \mathrm{umol} / \mathrm{L}$. The immunoglobulin profile showed $\mathrm{lgA} 80 \mathrm{~g} / \mathrm{L}, \mathrm{lgG} 4.15 \mathrm{~g} / \mathrm{L}, \mathrm{lgM} 0.04 \mathrm{~g} / \mathrm{L}$. The serum and urine immunofixation electrophoresis demonstrated IgA-Kappa monoclonal gammopathy and the $\beta 2$-microgloubulin was $8.89 \mathrm{mg} / \mathrm{L}$. Bone marrow aspiration showed monotypic plasma cells accounted for $60 \%$. The final diagnosis was Immunoglobin (Ig)A-K type Multiple Myeloma, International staging system (ISS) III, Durie-Salmon stage (D-S) IIIB. She had no significant medical history except myomectomy. She sequentially received BCD (Bortezomib plus cyclophosphamide and dexamethasone), TD (Thalidomide and dexamethasone), RD (lenalidomide and dexamethasone), RVD (lenalidomide plus bortezomib and dexamethasone), IRD (Ixazomib plus lenalidomide and dexamethasone), and BD (bortezomib and dexamethasone), while this patient suffered repeated disease progression due to the poor drug tolerance. In November 2019, this patient was diagnosed as r/rMM again. Daratumumab was initiated as a part of DVD regime (Daratumumab plus bortezomib and dexamethasone). Bortezomib was discontinued due to its side effects of intestinal obstruction and myelosuppression in January 2020. The patient's condition had been relieved to some extent. In May 2020 the disease progressed, impelling bortezomib to be recovered the fourteenth time. In 8 June 2020 when the frequency of daratumumab was reduced to once every four weeks, the patient developed bilateral pleural effusion (Fig. 1A). Diagnostic thoracentesis produced bloody fluid with features suggestive of a exudate (specific gravity 1.030 , nucleated cells count $11530 / \mathrm{mm}^{3}$, mononuclear cell $88 \%$, total protein $36.7 \mathrm{~g} / \mathrm{L}$, albumin $23.5 \mathrm{~g} / \mathrm{L}, \mathrm{LDH} 463 \mathrm{U} / \mathrm{L}, \mathrm{ADA} 40.1 \mathrm{U} / \mathrm{L}$ ). The microbiological examination including fungus and mycobacterium tuberculosis did not revealed pathogenic germ's growth and the Next Generation Sequencing (NGS)technology disclosed no pathogen possessing pathogenicity. Cytological analysis and flow cytometry analysis revealed large amount of abnormal plasma cells. In 9 June 2020 , the thoracic drainage was performed lasting 8 days in combination with DATD chemotherapy (Daratumumab, Liposomal Adriamycin, Thalidomide, and dexamethasone), during which the right pleural effusion presented a decreasing trend. The chemotherapy regime was replaced to PD (Pomalidomide and dexamethasone) and meanwhile there is a slowly increasing tendency of the left pleural effusion (Fig. 1B: 15 July 2020, chest CT). The patient ceased taking Pomalidomide as a result of myelosuppression in July 2020. The patient once again developed dyspnea in 17 August 2020 with chest CT (Fig. 1C) signifying the obvious increase of left pleural effusion and decrease of right pleural effusion. Diagnostic thoracentesis one more time produced exudative bloody fluid. The microbiological examination again got a negative result. Cytological analysis and flow cytometry analysis revealed large amount of abnormal plasma cells again. AD regime (Liposomal Adriamycin and dexamethasone) was chose together with respiratory support, left thoracic closed drainage and anti-infection therapy. There was a temporary improvement in the patient's condition. Chest CT was re-carried out in September (Fig. 1D, 3 September 2020), disclosing ground-glass opacity and consolidation in the right lung, diffuse ground-glass opacity in the left lung, the progression of lesions in upper lobe and decrease of bilateral pleural effusion. The patient died of exacerbation of pulmonary infection and respiratory failure on September 8, 2020. During the course of disease, the cytokine profile in pleural effusion and peripheral blood were detected by Biolegend CBA technology, which are showed in Table 1. 
Table 1

The cytokines concentrations are as follows:

\begin{tabular}{|c|c|c|c|c|c|c|c|c|c|c|c|c|}
\hline Time & Sample & $\begin{array}{l}\text { IL2 } \\
\mathrm{pg} / \mathrm{ml}\end{array}$ & $\begin{array}{l}\text { IL4 } \\
\mathrm{pg} / \mathrm{ml}\end{array}$ & $\begin{array}{l}\text { IL10 } \\
\mathrm{pg} / \mathrm{ml}\end{array}$ & $\begin{array}{l}\text { IL6 } \\
\mathrm{pg} / \mathrm{ml}\end{array}$ & $\begin{array}{l}\text { IL17A } \\
\mathrm{pg} / \mathrm{ml}\end{array}$ & $\begin{array}{l}\text { TNFa } \\
\mathrm{pg} / \mathrm{ml}\end{array}$ & $\begin{array}{l}\text { INFY } \\
\mathrm{pg} / \mathrm{ml}\end{array}$ & $\begin{array}{l}\text { Granzyme- } \\
\text { A } \\
\mathrm{pg} / \mathrm{ml}\end{array}$ & $\begin{array}{l}\text { Granzyme- } \\
\text { B } \\
\mathrm{pg} / \mathrm{ml}\end{array}$ & $\begin{array}{l}\text { Perforin } \\
\mathrm{pg} / \mathrm{ml}\end{array}$ & $\begin{array}{l}\text { Granulysin } \\
\mathrm{pg} / \mathrm{ml}\end{array}$ \\
\hline 2020.06 .09 & $\begin{array}{l}\text { Peripheral } \\
\text { blood }\end{array}$ & $<8.17$ & 1.48 & 85.23 & 12.00 & $<1.28$ & $<1.80$ & $<3.43$ & 20.88 & 9.13 & 986.97 & 655.00 \\
\hline 2020.06.09 & $\begin{array}{l}\text { Right } \\
\text { pleural } \\
\text { effusion }\end{array}$ & $<8.17$ & $<1.18$ & 1488.16 & 4884.91 & $<1.28$ & 2.37 & $<3.43$ & 57.52 & 721.32 & 1108.74 & 550.25 \\
\hline 2020.06 .15 & $\begin{array}{l}\text { Right } \\
\text { pleural } \\
\text { effusion }\end{array}$ & 1301.42 & 7.56 & 1.62 & 2685.95 & 795.89 & 22.75 & 15.22 & 319.46 & 1141.92 & 3517.97 & 1668.17 \\
\hline 2020.08 .17 & $\begin{array}{l}\text { Left } \\
\text { pleural } \\
\text { effusion }\end{array}$ & 15.05 & 0.83 & 1027.33 & 11088.80 & $<1.28$ & 5.54 & 9.32 & 100.27 & 435.53 & 243.55 & 945.15 \\
\hline
\end{tabular}

Abbreviations: IL-2: Interleukin 2; IL-4: Interleukin 4; IL-10: Interleukin 10; IL-6: Interleukin 6; IL-17A: Interleukin 17A; TNF-a: tumor necrosis factor a; INF-y: Interferon $y$.

\section{Discussion}

When MPE as the initial symptom of MM is easy to be misdiagnosed as tuberculous pleural effusion because both are exudative and around 1 / 3 of MPE also has a higher ADA level. ${ }^{1}$ Furthermore, a proportion of myeloma plasma cells are close to lymphocytes due to their intrinsic cytomorphological heterogeneity. The sensitivity of PPD test, T-spot. TB, mycobacterium tuberculosis culture, PCR and NGS technology to make the diagnosis of tuberculosis pleural effusion is comparatively low. ${ }^{7}$ While the sensitivity of pleural biopsy is satisfying, its invasive nature imposes restriction on its application. Although ADA has both high sensitivity and specificity, ${ }^{7}$ it is not suitable to be a differentiating criterion due to its increased level in MPE. The sensitivity and specificity of INF-y are reported as high as $89-99 \%$ and $92-98 \%$ respectively. As a distinctive cytokine of Th1 cells, INF-y in peripheral blood and pleural effusion are significantly elevated by activated macrophages after the infection. Nevertheless, this patient was immunosuppressed, meanwhile INF- $y$ both in peripheral blood and pleural effusion were below the detectable range. There may be a great difference of INF-y level between MPE and tuberculous pleural effusion, which draw a large prospect to apply it to make the identification.

MPE is usually an advanced manifestation of MM and its median survival time is only 2.8-4.0 months even under active treatment. ${ }^{1}$ At present, multi-drug chemotherapy combined with repeated thoracocentesis drainage or pleurodesis is strongly recommended. ${ }^{1}$ The right pleural effusion of this patient was significantly reduced after the application of DATD regimen combined with thoracocentesis drainage. Moreover, no relapse occurred under maintenance treatment of pomalidomide alone. We revealed that the level of IL-2, IL-4, IL-17A, TNF - $a$, INF - $\gamma$, granzyme A, Granzyme B, perforin and granulysin in pleural effusion elevated, while the cytokines IL- 6 and IL-10 related to the tumor load significantly decreased in the course of DATD regime, which indicates the level of cytokines in pleural effusion is closely related to disease deterioration and improvement. The detection of cytokines in pleural effusion may become a barometer to predict the variation tendency of pleural effusion.

IL-2 is an important immunoregulatory cytokine in various diseases. ${ }^{8} \mathrm{~A}$ previous study reported that the chemotherapy combined with IL-2 immunotherapy showed good therapeutic effect in 2 cases of MPE with survival time as long as 30 months. ${ }^{4}$ Our patients showed a higher IL-2 concentration in remission of pleural effusion and a lower IL-2 level in relapse. In the light of IL-2 can down-regulate the expression level of PD-1 and promote the secretion of granzyme B and IFN - $y$ to enhance tumor killing ability, ${ }^{8}$ it is considered to be able to reverse the exhaustion of T cell, which is probably related to its positive therapeutic effect. In our case, Granzyme B and IFN - y in pleural effusion increased with the increase of IL-2, which fully verified this mechanism. If T cell exhaustion reversing is the main mechanism of IL-2 in therapy, PD-1 inhibitor perhaps has a good prospect as well, which requires further research. Based on the disease course of this patient, the immunoregulators (Thalidomide and Pomalidomide) are suspected to lead to cytokines changing to a large extent.

Immunomodulators, including thalidomide, lenalidomide and Pomalidomide, can induce T lymphocytes to secrete IL-2 through CRBN signaling pathway, and further inhibit the proliferation of myeloma cells, ${ }^{9}$ which is capable to state the reason why IL-2 returned to the initial level and also the pleural effusion significantly progressed as the discontinuation of immunoregulators. Therefore, to regulate the immune situation in thoracic cavity constitute the possible mechanism to inhibit the formation of pleural effusion.

\section{Conclusion}

There is still no report depicting the important role of cytokines in pleural effusion until now. Our case report certify a foreground for the detection of INF-y to be employed to differentiate tuberculous pleural effusion from MPE patients. In addition, the concentrations of various cytokines in pleural effusion are potential barometers to forecast the variation tendency of pleural effusion. Finally, our work may turn into the stepping stone for immunothrapy to participate in therapy of MPE.

\section{Declarations}

\section{Acknowledgments}


We thank his close relatives for allowing us to publish his case.

\section{Authors' contributions}

JHX and LG participated in the acquisition of clinical data and wrote the manuscript. MY, BJW, ZYS, HHL, KYS, and LN carried out the histological examination and interpretation. MJW and XNC revised the manuscript. All authors have read and approved the final manuscript.

\section{Funding}

This study was funded by the Beijing Municipal Science Technology Commission (No. Z191100006619026). The funders had no role in the study except for financial support.

\section{Availability of data and materials}

All data generated or analysed during this study are included in this published article.

\section{Ethics approval and consent to participate}

Not applicable.

\section{Consent for publication}

Written informed consent for publication was obtained from the patient's relatives.

\section{Competing interests}

The authors declare that they have no conflict of interest.

\section{References}

1. Yanamandra U, Deo P, Sahu KK,Nampoothiri RV,Gupta N, Prabhakaran A,et al. Clinicopathological Profile of Myelomatous Pleural Effusion: Single-center Real-world Experience and Review of Literature. Clinical lymphoma myeloma leukemia. 2019;19:183-9.e1.

2. Kintzer JS, Jr.,Rosenow EC, 3rd,Kyle RA. Thoracic and pulmonary abnormalities in multiple myeloma. A review of 958 cases. Arch Intern Med. 1978;138: $727-30$.

3. Cho YU, Chi HS,Park CJ, Jang S, Seo EJ,Suh C. Myelomatous pleural effusion: a case series in a single institution and literature review. Korean J Lab Med. 2011;31:225-30.

4. Zhong $\mathrm{Y}$, Zhang J, Wang $\mathrm{H}$. Myelomatous pleural effusion involvement in 23 patients with multiple myeloma: A single-center clinical analysis. Thorac Cancer. 2015;6:359-62.

5. Makino S, Yamahara S, Nagake Y, Kamura J. Bence-Jones myeloma with pleural effusion: response to alpha-interferon and combined chemotherapy. Intern Med (Tokyo Japan). 1992;31:617-21.

6. Riveiro V, Ferreiro L, Toubes ME, Lama. A,Álvarez-Dobaño JM,Valdés L. Characteristics of patients with myelomatous pleural effusion. A systematic review. Revista clinica espanola. 2018;218:89-97.

7. Villena Garrido V, Cases Viedma E, Fernández Villar A, de Pablo Gafas A,Pérez Rodríguez E,Porcel Pérez JM,et al. Recommendations of diagnosis and treatment of pleural effusion. Update. Archivos de bronconeumologia. 2014;50: 235 - 49.

8. Han L, Jiang Q, Yao W, Fu T, Zeng Q. Thoracic injection of low-dose interleukin-2 as an adjuvant therapy improves the control of the malignant pleural effusions: a systematic review and meta-analysis base on Chinese patients. BMC Cancer. 2018;18:725.

9. Lindner S,Krönke J. The molecular mechanism of thalidomide analogs in hematologic malignancies. 2016;94: $1327-34$.

\section{Figures}




\section{Figure 1}
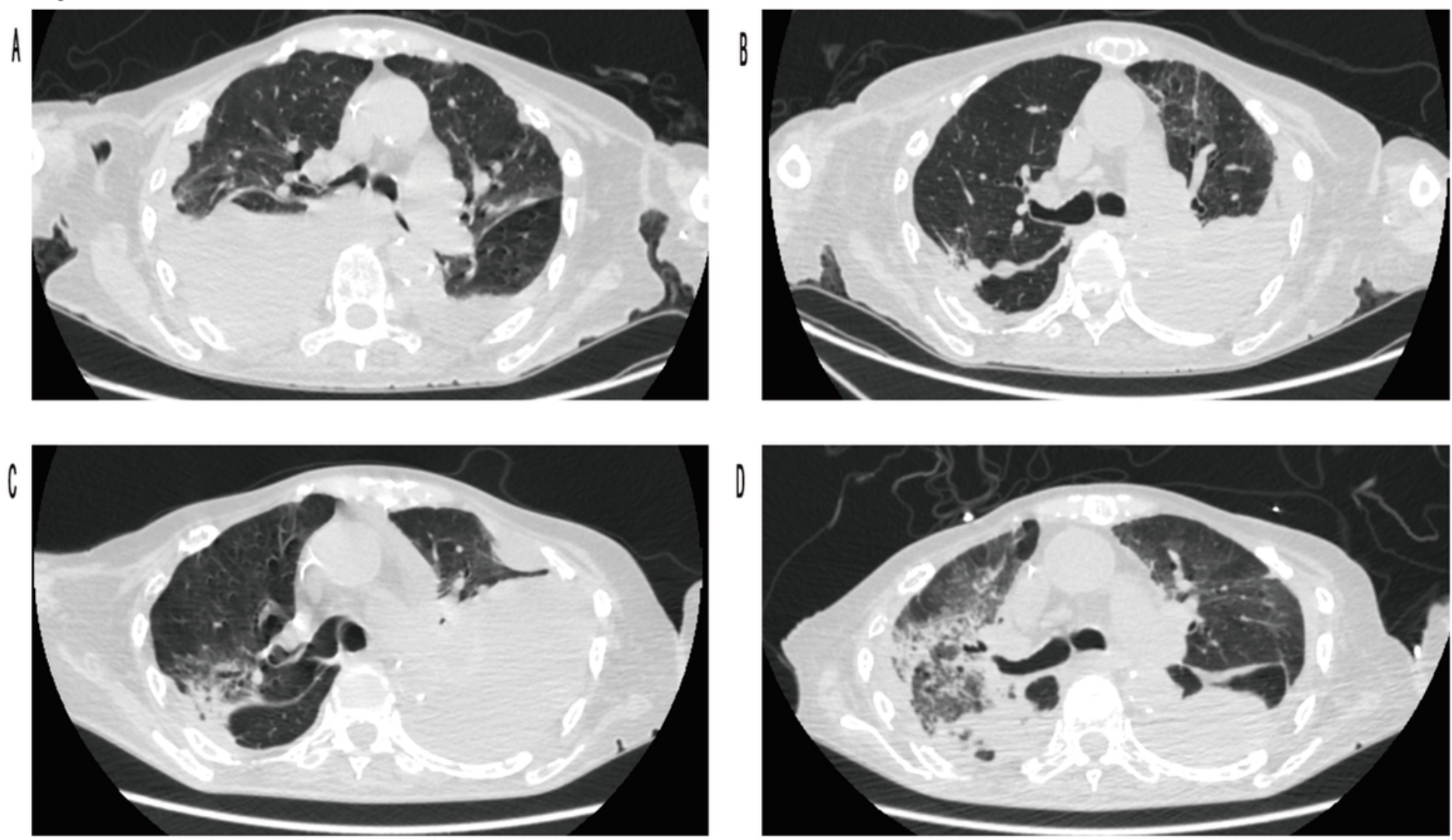

Figure 1

Changes in chest CT findings during the course of the disease. A. Initial stage: Chest CT showed bilateral pleural effusion as well as left interlobar effusion and no manifestation of acute inflammation and space-occupying lesions. B. Remission stage: slowly increasing tendency of the left pleural effusion and no increasing in right pleural effusion. C. Relapse stage: obvious increase of left pleural effusion and decrease of right pleural effusion. D. Terminal stage: Ground-glass opacity and consolidation in the right lung, diffuse ground-glass opacity in the left lung, the progression of lesions in upper lobe and decrease of bilateral pleural effusion.

\section{Figure 2}

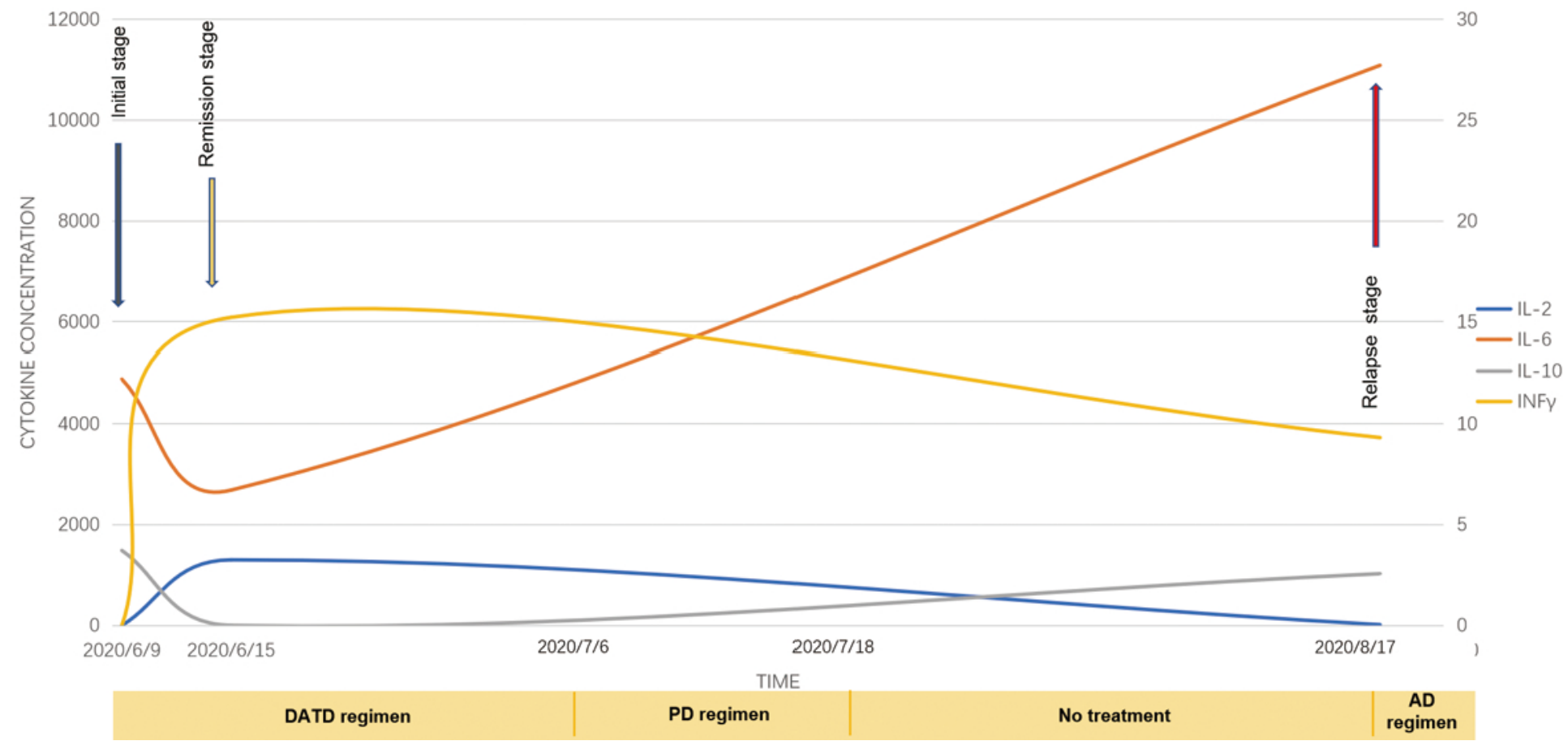


Figure 2

The concentrations of IL2, IL6, IL10, INF-y at initial stage, remission stage, and relapse stage during the course of the disease. Abbreviation: DATD: Daratumumab, Liposomal Adriamycin, Thalidomide and dexamethasone; PD: Pomalidomide and dexamethasone; AD: Liposomal Adriamycin and dexamethasone. 